\title{
Enquête sur l'évaluation de l'anglais par les Anglophones dans les publications des scientifiques français
}

Critères linguistiques, résultats et perspectives

\section{Elizabeth Crosnier}

\section{OpenEdition}

\section{Journals}

Édition électronique

URL : http://journals.openedition.org/asp/4229

DOI : $10.4000 /$ asp. 4229

ISSN : 2108-6354

\section{Éditeur}

Groupe d'étude et de recherche en anglais de spécialité

\section{Édition imprimée}

Date de publication : 1 février 1994

Pagination : $39-55$

ISSN : 1246-8185

Référence électronique

Elizabeth Crosnier, «Enquête sur l'évaluation de l'anglais par les Anglophones dans les publications des scientifiques français », ASp [En ligne], 3 | 1994, mis en ligne le 19 février 2014, consulté le 19 avril 2019. URL : http://journals.openedition.org/asp/4229 ; DOI : 10.4000/asp.4229

Ce document a été généré automatiquement le 19 avril 2019.

Tous droits réservés 


\title{
Enquête sur l'évaluation de l'anglais par les Anglophones dans les publications des scientifiques français
}

Critères linguistiques, résultats et perspectives

\author{
Elizabeth Crosnier
}

1 Cent-vingt questionnaires accompagnés d'une lettre d'explication ont été adressés à des referees ou reviewers au Canada, aux États-Unis et au Royaume-Uni. Ces destinataires, qui travaillent pour le compte de revues scientifiques anglophones, étaient alors invités à fournir des informations sur la manière dont ils évaluent la qualité de l'anglais des articles produits par des chercheurs français avant publication, et les critiques qu'ils émettent à leur égard. L'objectif de l'enquête est de savoir ce que les anglophones attendent des chercheurs français en matière de publication scientifique du point de vue linguistique puisque la langue semble être un handicap parfois important. Nous présenterons donc des évaluations tant qualitatives que quantitatives à partir des données recueillies, puis une synthèse des commentaires. Enfin en conclusion, après un bilan global, nous montrerons l'intérêt d'explorer le monde de la publication scientifique.

\section{Présentation de l'enquête}

2 L'enquête qui va être présentée dans cet article fait suite à une étude menée en 1990 (Crosnier) dont l'objectif était de quantifier les besoins en anglais d'ingénieurs, techniciens et chercheurs de langue française, et ensuite d'estimer leurs problèmes consécutifs à une utilisation plus ou moins intensive selon les disciplines, les environnements et les activités. Cette première enquête sous forme de questionnaire écrit a été le point de départ d'une analyse de leurs difficultés en anglais dans le cadre de leurs activités professionnelles. Les résultats obtenus exprimaient les points de vue de 120 francophones de douze disciplines scientifiques et techniques. Ceux-ci seront brièvement rappelés dans la conclusion afin de rapprocher ces deux études 
complémentaires. Ces enquêtes s'inscrivent dans un vaste projet de recherche sur l'anglais scientifique et ses implications, intégrant une analyse stylistique du discours et les processus de traduction mis en œuvre.

Après avoir tenté de cerner et d'évaluer les problèmes de personnes de langue française, nous avons pensé qu'il serait probablement intéressant, et utile, de connaître les exigences des lecteurs anglophones. En effet, il est nécessaire de rappeler une difficulté majeure rencontrée actuellement par les scientifiques francophones, et par conséquent de justifier les démarches entreprises et l'étude qui va en découler. Un premier constat s'impose à la suite de nombreux entretiens : ceux qui tentent de publier dans des revues anglophones se heurtent fréquemment à un barrage d'ordre linguistique. Leurs articles leur sont souvent retournés avec des remarques telles que " poor English", accompagnées parfois d'une liste d'erreurs plus ou moins graves, de commentaires désobligeants sur la qualité du langage, voire un refus catégorique sans offrir à l'auteur la moindre possibilité de révision. Voici quelques extraits de rapports :

Despite the very poor copy of xxs paper "An introduction to Schoenberg's Approximation" which I have received, I have made some attempt to read it and to advise you on its suitability for the special issue.

However, you must not allow this paper to be published in its present form. There are many misprints, sentence fragments, grammatical errors, and some sentences that are positively unintelligible. I suggest, for example, that you turn to page 5 and read the description of Martin Buhmann's work.

Of course the author is French and so I sympathise with his efforts. But in order that the paper be usable, it will need extensive editorial work.

However some questions should be pointed out the author before publication, due to the feeling that he has not sufficiently revised the typed text before submitting it.

- A lot of commas, periods, etc. have been omitted between formulas and lines, as I have marked on the manuscript not $m$ an exhaustive way.

- The English text in Section 6 presents, in some parts, misprints or mistakes, as consequences (for consequences), convex envelope (for convex hull), total positive (for totally positive) and so on. The style should be improved in this section, with frequent repetitions as "As it is known" in two consecutive lines, etc.

This is an interesting paper which throws light on the interaction of the magnetization in adjacent grains in this important material. It is rather difficult to read as it stands and I suggest the authors revise the English along the lines I have indicated. Then it will be a useful contribution to the conference proceedings.

4 La langue représente assurément un obstacle, avant même le contenu purement scientifique, ce qui provoque quelquefois des malentendus et des préjugés exprimés avec amertume ou colère par les chercheurs. De plus, certains ressentent parfois ce handicap comme une volonté délibérée de la part des anglophones de bloquer leur accès à la diffusion internationale. C'est pourquoi nous avons voulu y voir plus clair malgré la complexité de la situation et l'accès difficile à un monde fermé. Il nous est permis d'avancer aussi deux autres hypothèses. La langue peut servir d'excuse pour refuser des articles scientifiquement "insuffisants» ou d'autre part, pour faire barrage lorsque lesdits articles ne sont pas « souhaitables " pour des raisons par exemple de compétitivité ou de priorité.

5 Deux catégories d'interlocuteurs ont donc été sélectionnées: d'une part les chercheurs francophones qui souhaitent publier leurs articles dans des revues anglaises, canadiennes ou américaines, d'autre part les réviseurs, spécialistes scientifiques responsables de l'évaluation et de la révision des articles avant publication. Les deux publics ciblés sont 
mis en confrontation régulièrement, car l'accès à ces publications est actuellement l'objectif des chercheurs français de haut niveau. C'est à la lecture de quelques rapports et après avoir entendu certains témoignages que l'idée a surgi. La situation semblait alors confuse : manque de données précises, des contradictions apparentes et des remarques mal perçues. Par conséquent, en plus des exigences, il paraissait indispensable de découvrir les critères d'évaluation s'ils existaient et s'ils étaient clairement définis, et éventuellement les impressions ou critiques de ces « juges » de langue anglaise à l'égard des auteurs français. Ce dernier point est essentiel, car il peut en réalité être déterminant. Toutefois, il devra être traité avec beaucoup de prudence contrairement à l'évaluation quantitative puisqu'il s'agit d'interpréter des mots et de percevoir le non-dit à travers les commentaires.

Cependant, s'il est relativement facile d'obtenir ces informations grâce au questionnaire, il est beaucoup plus difficile d'en vérifier la crédibilité nécessaire pour une analyse complète de la situation. Le seul moyen de contrôler la bonne application des règles et conditions requises, ou déclarées, est l'accès aux rapports produits par ces mêmes réviseurs, ce qui est actuellement laborieux ; cinq seulement nous ont été fournis dont nous avons donné un aperçu plus haut, ce qui n'est pas suffisant pour l'analyse. Cette piste devra être abandonnée et nous serons amenés à limiter notre champ d'investigation. L'objectif est alors de présenter et de commenter les résultats de cette seconde enquête. En supposant que ces informations sont crédibles et peuvent servir de références, nous proposerons un double bilan: l'un sur les conditions actuelles de publication en pays anglophone pour des non-anglophones, l'autre sur la concordance entre les discours des deux parties.

\section{Organisation}

7 Les réviseurs interrogés opèrent dans les circuits les plus diversifiés : milieu universitaire ou industriel, centres de recherches, académies ou sociétés, en Grande-Bretagne, au Canada et aux États-Unis, et ils sont tous responsables de la révision d'articles soumis à publication pour le compte de revues ou périodiques à diffusion internationale disponibles dans les bibliothèques spécialisées. Les domaines sélectionnés sont les mathématiques, la mécanique et l'informatique, notre travail portant essentiellement sur ces disciplines. Voici les contraintes que nous nous sommes imposées :

- public concerné : referees ou technical editors anglophones responsables de la révision d'articles avant publication ;

- revues britanniques ou américaines à diffusion internationale ouvertes aux chercheurs du monde entier ;

- diversité des revues : publications pour des universités, laboratoires de recherche, sociétés, ou revues indépendantes;

- disciplines considérées : mathématiques, mécanique et informatique ;

- diversité géographique.

8 Quarante-six réponses nous sont parvenues avant la date fixée pour le dépouillement, ce qui donne un pourcentage de 38,33 . Les quelques réponses reçues après cette date n'ont pas été exploitées, elles n'auraient d'ailleurs pas modifié les résultats de manière significative. Ce score est satisfaisant étant donné les conditions, c'est-à-dire pour des destinataires étrangers dont les activités et motivations sont bien différentes des nôtres, 
abordant une situation délicate plus ou moins teintée de polémique, et ceci sans lettre de relance avec un risque de rejet élevé.

\section{Objectifs}

Nous espérions des réponses aux interrogations suivantes:

- Qu'attendent les éditeurs de revues anglophones des auteurs français d'articles scientifiques et leurs exigences sont-elles explicites?

- Ont-ils élaboré une grille où les critères linguistiques sont clairement définis?

- Les exigences et les critères varient-ils selon les disciplines ou les nationalités?

- Les chercheurs peuvent-ils espérer une certaine aide, ou collaboration, de la part des responsables d'édition?

10 Nous avons dit précédemment que la situation semblait confuse. Nous souhaitions alors nous appuyer sur des données précises et non plus traiter le problème de façon empirique, l'objectif à plus long terme étant de fournir aux chercheurs de langue française une grille d'informations qui les aiderait à mieux rédiger leurs articles en répondant aux exigences des revues. En effet, grâce aux remarques notées et aux critères mis en valeur, aux documents fournis pour l'aide à la rédaction et à l'analyse approfondie des écrits scientifiques en anglais, nous pensons être en mesure de concevoir une documentation sur l'usage de l'anglais à l'intention des scientifiques français. Ceci aurait, même modestement, des effets bénéfiques en matière de gain de temps en évitant des retours et de longues révisions, et à la limite, des refus catégoriques de publication, tout en stimulant la production. Ces données pourraient être également exploitées en amont pour la formation en langues destinée aux étudiants futurs chercheurs.

11 Enfin, les informations et les documents fournis par les réviseurs permettent de déterminer en partie les spécificités du langage scientifique, en partie seulement puisque nous traitons d'un discours précis, celui des articles de revues. Ces caractéristiques seront utilisées comme outil de référence pour l'analyse stylistique de l'anglais de spécialité actuellement en cours.

\section{Résultats}

Le questionnaire a été formulé de manière à obtenir les informations souhaitées sans abuser de la bonté des destinataires dont on connaît l'indisponibilité et les motivations très sélectives. C'est pourquoi le texte est court et concis. Seuls les commentaires peuvent compléter ou nuancer les réponses. Les questions fermées 1,2 et 5 portent sur les conditions d'évaluation, les questions 3 et 4 sur les critères linguistiques. Les commentaires éventuels viennent en complément.

13 Le questionnaire proposé figure en annexe.

\subsection{Questions 1,2 et 5}

Les histogrammes représentant les données en chiffres réels sur 46 réponses aux questions 1 (Do you have general guidelines for assessing research papers?) et 2 (If so, are there specific criteria concerning the quality of the English idiom?) sont donnés à la figure 1. 
Figure 1. Histogramme question 1 et question 2
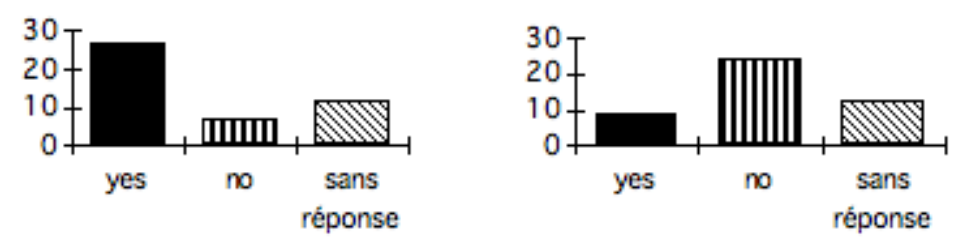

15 Les questions 1 et 2 sont des questions totalement objectives sur la mise en place du système d'évaluation des articles. Les histogrammes représentant le nombre de réponses sont éloquents. Si plus de la moitié des réviseurs ont à leur disposition une grille générale plus ou moins semblable à celles présentées sur la page suivante, très peu d'entre eux, 1/5 seulement, ont une rubrique concernant la qualité de l'anglais, qui pourrait être d'ailleurs valable pour les anglophones. Remarquons en confrontant les deux histogrammes que les résultats s'inversent. Nous présentons ci-dessous quelques extraits de grilles d'évaluation avec l'autorisation des revues.

16 Le nombre de sans réponses est élevé, surtout à la question 2, question apparemment gênante, et peut être assimilé sans trop de risque à des réponses négatives, ce qui prouverait que de nombreuses revues n'établissent pas de grille définie. Par conséquent, il semble que leurs réviseurs évaluent les articles sans critères précis. Cet état de fait laisse supposer que ceux-ci peuvent exprimer leurs impressions personnelles en fonction de leurs objectifs, de leur sensibilité ou même d'autres paramètres. On imagine difficilement une évaluation indépendante de toute perception individuelle. C'est sans doute pour cette raison que les articles sont révisés par deux personnes à la fois. Nous essayerons de chercher au travers des commentaires une confirmation de cette hypothèse.

17 La question 5 (Do you have an "Anglicizing” service for correction?) n'a été formulée qu'au deuxième envoi, concernant ainsi 26 personnes dont huit ont répondu " yes » et 15 "no ». On constate qu'un nombre non négligeable, presqu'un tiers des revues prévoient de corriger les erreurs de langue, ce qui traduit un certain désir de coopération. Les commentaires nous apporteront quelques précisions sur le type de fautes corrigibles par ce service et les limites qu'il s'impose éventuellement, mais il est difficile de savoir si en pratique, les articles sont réellement corrigés.

\subsection{Questions 3 et 4}

18 Les questions 3 (What are the linguistic criteria taken into account concerning the papers written in English by French scientists?) et 4 (What errors among the above-mentioned points are always unacceptable?) ont pour objectif de déterminer les critères linguistiques considérés par les réviseurs, et l'importance qu'ils y attribuent. La question 3 est présentée sous forme de tableau et de cases à cocher. La question 4 est semi-ouverte et complète la précédente.

\section{Présentation du tableau - question 3}

19 Nous avons répertorié huit variables correspondant aux critères qui interviennent dans la formulation du discours : 2 pour le vocabulaire, 2 pour l'aspect grammatical, 3 pour l'organisation du discours et 1 pour l'aspect idiomatique. Pour chaque variable nous 
avons établi 3 modalités, 2 extrêmes avec "always" et "never » et 1 intermédiaire " sometimes». La $4^{\mathrm{e}}$ modalité correspond au nombre de personnes n'ayant pas fourni de réponses. Dans ce questionnaire, nous n'avons retenu que des critères généraux avec une terminologie simple afin de ne pas embarrasser les réviseurs. Il nous semblait préférable de perdre en nuances plutôt que de risquer un rejet.

\section{Résultats - question 3}

Dans cette partie, nous essayerons de mettre en valeur les aspects les plus flagrants et nous émettrons quelques hypothèses que seule l'étude des commentaires pourra confirmer. Les histogrammes permettent de visualiser rapidement les résultats. Chaque colonne correspond à une des huit variables du questionnaire, les réponses aux quatre modalités sont superposées et les résultats sont exprimés en pourcentages.

Figure 2. Histogramme question 3

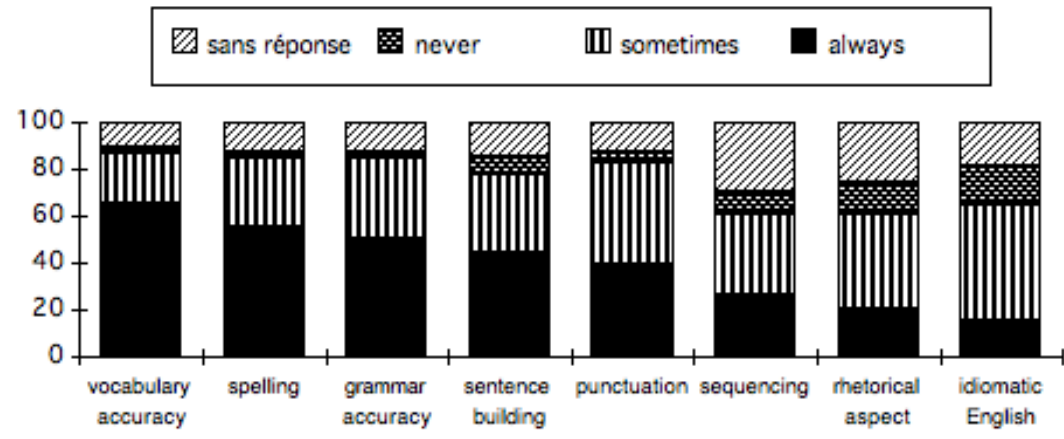

21 Pour la modalité «always", la variable qui se rapporte au vocabulaire obtient le pourcentage le plus élevé. Notons le mouvement décroissant vers l'anglais idiomatique avec une légère inversion seulement au niveau des variables 5 et 6 . En revanche, le mouvement est inverse pour la modalité «never». Ces deux remarques montrent la prépondérance qu'attribuent la plupart des enquêtés au vocabulaire, avec l'orthographe, dans le langage scientifique. On voit également l'intérêt que portent les moins catégoriques à ce même critère avec les réponses à la modalité « sometimes ».

Les réviseurs attribuent également une grande importance à l'aspect grammatical. La phrase doit être juste du point de vue lexical et grammatical avec une ponctuation utilisée correctement afin de rendre le message compréhensible. Les autres éléments qui interviennent dans le discours sont secondaires.

23 Le nombre de personnes n'ayant pas répondu aux variables 1 à 5 est faible et constant. En revanche il augmente nettement pour les trois dernières variables. Il est toujours difficile d'interpréter les taux de "sans réponses ». Ici, nous nous risquons à exprimer quelques hypothèses :

- manque de motivation pour le sujet ;

- révision opérée de manière intuitive sans analyse préalable des critères ;

- aucune importance attribuée au langage ;

- absence de réflexion.

Quelle que soit la raison, ces résultats sont encore trop élevés s'ils correspondent à un manque de fondement du système de révision. De plus, ajoutés aux résultats obtenus pour la modalité «never ", on atteint des pourcentages élevés surtout pour les variables 6 et 7 . Ces critères ont laissé les réviseurs perplexes, les points d'interrogation remplaçant 
parfois les croix confirment cette indétermination. On peut croire que le sens des mots « sequencing " et "rhetorical » n'est pas clair pour tous les scientifiques, que ces derniers n'en voient pas l'application, ou bien encore qu'ils ne se sont pas interrogés en tant que natifs sur des concepts qu'ils ont intériorisés sans les formuler consciemment.

C'est à ce propos que se présente le problème de la « conscience linguistique » qu'a une personne de sa propre langue : est-il possible d'émettre des jugements sur les énoncés émis dans cette langue, rôle qui est assigné aux réviseurs?

En additionnant les chiffres obtenus aux modalités «always» et «sometimes " pour les variables 1 à 5 , on obtient un nombre stable, de 40 à 38 , et de même pour les variables 6 à 8,28 à 30. Ces données confirment le peloton de tête des critères qui garantissent la qualité de la phrase anglaise: un vocabulaire juste et des structures grammaticales correctes.

\section{Résultats - question 4}

La question 4 (What errors among the above-mentioned points are always unacceptable?) a été posée afin d'affiner l'enquête et la compléter. Elle a pour but de confirmer les résultats présentés ci-dessus. Le nombre de réponses obtenues figure dans le tableau 1.

Tableau 1

\begin{tabular}{|l|l|}
\hline Variable & Nombre de réponses \\
\hline 1 & 13 \\
\hline 2 & 11 \\
\hline 3 & 9 \\
\hline 4 & 9 \\
\hline 5 & 4 \\
\hline 6 & 3 \\
\hline 7 & 2 \\
\hline 8 & 0 \\
\hline
\end{tabular}

28 Ces chiffres confirment effectivement les résultats précédents, mais ils sont peu élevés. Faut-il les interpréter comme une preuve de tolérance? On imagine que le jugement à l'égard de certaines fautes n'est pas aussi sévère que s'il émanait par exemple de linguistes. Mais peut-être que cette question n'a pas été jugée intéressante ni utile.

\subsection{Conclusions}

La justesse du vocabulaire est le point considéré comme primordial par les réviseurs avec l'orthographe ainsi que l'aspect grammatical. En revanche, ils sont apparemment moins exigeants sur l'organisation des idées dans la phrase, puis dans le paragraphe, et l'articulation du discours. Ils semblent faire preuve de grande tolérance à l'égard de 
l'aspect idiomatique. Ceci reflète la position générale, toutes nationalités et disciplines confondues. Au dépouillement, nous avons constaté des disparités entre les différents groupes, c'est pourquoi nous présentons ci-dessous une étude comparative. Notons toutefois quelques exceptions intéressantes mais peut-être peu représentatives: 3 personnes ont répondu «always » pour toutes les variables. Il s'agit d'un spécialiste de mécanique américain, d'un mathématicien canadien et d'un informaticien d'oxford. D'autre part, un mathématicien de Coventry, UK, a répondu «never » à toutes les variables.

\section{Analyse comparative des résultats}

Cette partie est consacrée à la comparaison des données recueillies pour la question 3 par discipline et par pays illustrée par des histogrammes exprimés en pourcentages. Il s'agit d'observer les réactions des réviseurs en fonction de ces paramètres et les écarts par rapport à la grille générale.

\subsection{Analyse par discipline}

Les réviseurs n'attribuent pas systématiquement la même importance aux différents critères et les histogrammes présentés ci-dessous mettent en évidence des divergences.

Figure 3. Mathématiques (21 réponses)

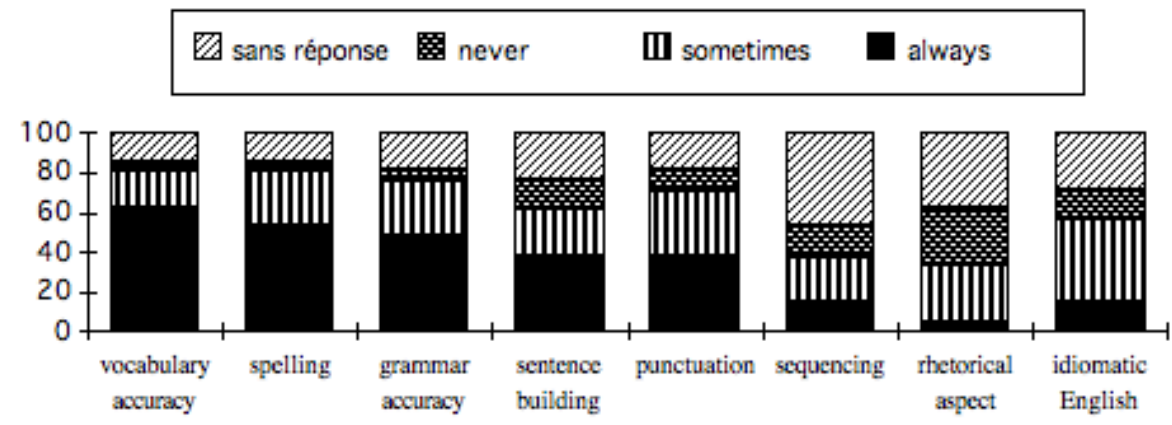

L'histogramme représentant les résultats obtenus auprès des mathématiciens est très proche de la moyenne générale et le profil des courbes est assez identique à l'exception des variables 6 et 7 où on note quelques écarts. Les pourcentages de réponses à la modalité "always" sont nettement plus faibles et le nombre de personnes n'ayant pas répondu est élevé surtout pour la variable «sequencing». Cette réaction est surprenante. La notion n'était peut-être pas claire, à moins qu'elle ne soit considérée comme une donnée acquise, mais non explicitée, due au fait que ces mathématiciens de haut niveau ont un raisonnement tellement structuré que l'enchaînement des parties du discours est inévitablement rigoureux. Et pourtant un chercheur américain de renom, Micchielli, n'hésite pas à interrompre ses démonstrations par des définitions, celles-ci intervenant donc à tout moment du discours comme des "flash-back», ce qui nécessite d'éternels mouvements de va-et-vient de la part du lecteur et par conséquent un langage modifié en fonction de ce mode de raisonnement. 
Figure 4. Informatique (15 réponses)

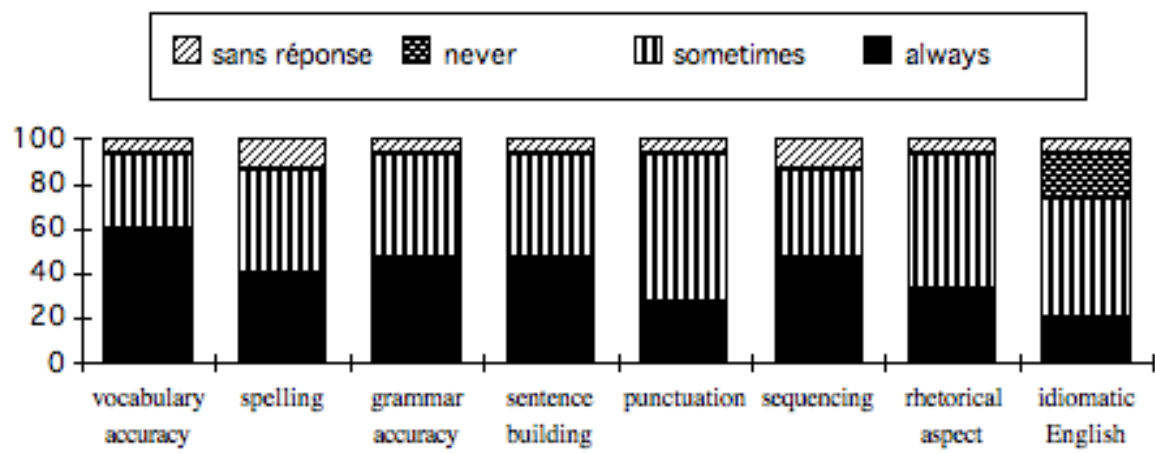

Les informaticiens ont des positions plus intermédiaires traduites par une majorité de " sometimes", aucune réponse "never» hormis à la variable 8 et peu de "sans réponse ». Notons aussi que le vocabulaire et l'orthographe ont une importance moindre ainsi que la ponctuation. Même l'aspect grammatical semble moins essentiel. En revanche, l'ordonnancement est davantage considéré ainsi que l'aspect idiomatique. Les variables 6 et 7 les ont peu embarrassés. Nous pouvons émettre l'hypothèse que les informaticiens sont imprégnés du langage de la machine, langage largement constitué de jargon, dont la syntaxe a été modifiée et le vocabulaire créé ou transformé. La langue informatique n'a pas d'histoire ni de modèle linguistique, d'où peut-être ces libertés prises par rapport à ce qu'on pourrait appeler un anglais de référence. L'enchaînement des fonctions dans la mise en place des programmes informatiques nécessite également une chronologie et un ordonnancement rigoureux, ce qui expliquerait l'importance attribuée à la modalité 6 . Une étude portant sur des textes informatiques menée en parallèle nous a déjà permis de vérifier un certain « laxisme » par rapport à l'anglais académique.

Figure 5. Mécanique (10 réponses)

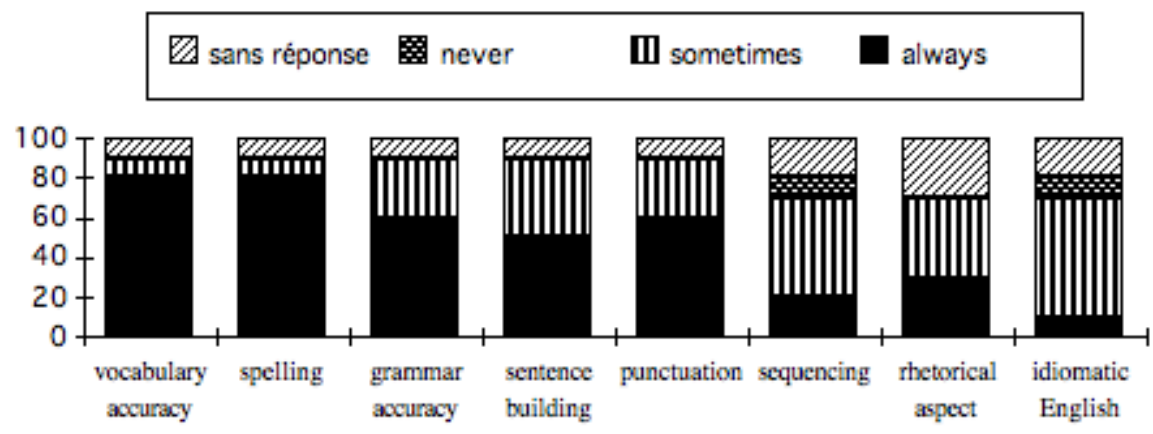

Le nombre de questionnaires remplis est plus faible que pour les autres disciplines, mais les réponses sont intéressantes. Les spécialistes de mécanique contrastent par leur rigueur. Cet aspect a d'ailleurs été vérifié à travers l'analyse d'un corpus de textes. Les réponses à la modalité « always » sont largement majoritaires pour les variables 1 à 5 avec des pourcentages élevés. Elles sont également supérieures à la moyenne pour les variables 6 et 7. Elles sont conformes à la moyenne seulement pour la variable 8 . Notons également l'absence de réponses «never». Si le passé du langage informatique est limité, la mécanique a une longue histoire qui a forgé une langue empreinte de traditionalisme, hypothèse qui peut être retenue pour expliquer ce désir de rigueur. 


\subsection{Analyse par pays} représentés montrent quelques points de vue divergents.

Figure 6. Grande-Bretagne (22 réponses)

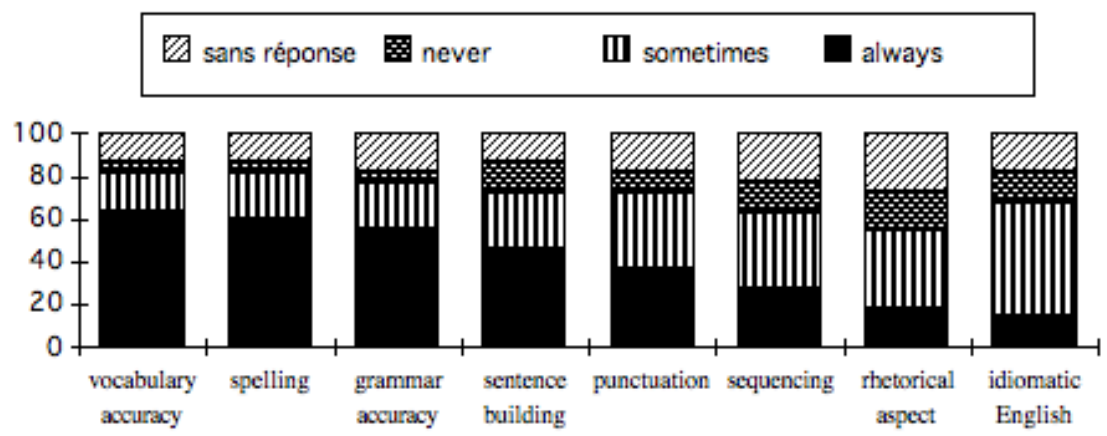

L'histogramme le plus proche de la moyenne est celui qui représente la Grande-Bretagne. Les différences sont insignifiantes. Nous remarquerons seulement un taux globalement plus élevé de « sans réponses » et une indifférence plus notoire pour la rhétorique.

Figure 7. États-Unis (20 réponses)

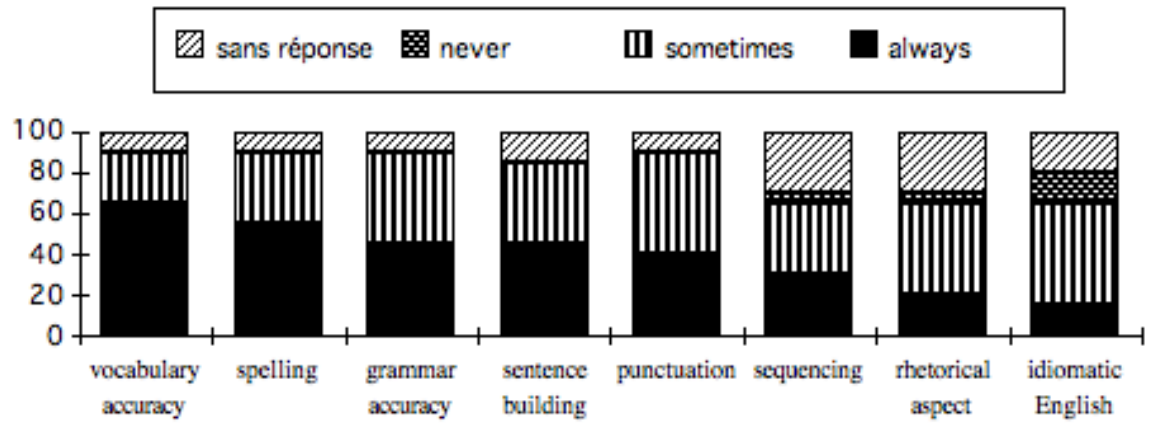

Pour les États-Unis, la courbe descendante qui schématise les réponses à la modalité « always » a le même profil que sur l'histogramme général, mais les pourcentages sont tous inférieurs. Cet élément semble prouver une exigence moindre de la part des Américains. Cette hypothèse reste à vérifier. Étant donné la difficulté qu'ont les scientifiques français à publier aux États-Unis, il serait nécessaire d'approfondir l'analyse afin de découvrir si d'autres facteurs interviennent au cours du filtrage des publications. Notons aussi l'absence de réponses à la modalité «never » pour les variables 1 à 5 .

\section{Canada (4 réponses)}

Malgré le chiffre réduit de réponses en provenance du Canada, nous avons tenu à présenter les résultats à titre d'information puisque l'échantillon n'est pas représentatif. Les critères essentiels sont le vocabulaire, la grammaire et la ponctuation. L'ordonnancement des idées n'est pas pris en considération. 


\subsection{Bilan}

En conclusion, nous établissons un rapprochement entre les Britanniques et les mathématiciens d'une part, les Américains et les informaticiens d'autre part, les premiers exigeant plus de rigueur sur la qualité de la langue. L'anglais idiomatique prend une place plus importante dans l'évaluation des réviseurs informaticiens. Les spécialistes de mécanique semblent être plus stricts que les représentants des autres groupes.

Nous avons voulu mettre en valeur quelques-uns de ces résultats. L'histogramme présenté plus loin correspond au croisement de la modalité « always » avec trois variables caractéristiques : le vocabulaire, la grammaire et l'aspect idiomatique, ceci pour chaque discipline et pour les deux pays les plus représentés. Les données sont exprimées en pourcentages par rapport à l'effectif total pour chaque catégorie. Le second graphique représente les courbes correspondant à chacune des trois variables.

Figure 8. Graphiques croisement avec la modalité always

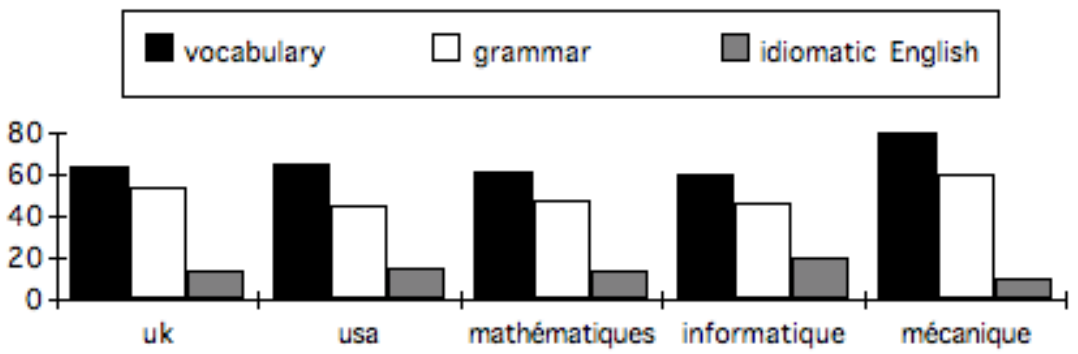

41 Ces graphiques mettent en évidence les données essentielles : on constate la stabilité de la courbe représentant le vocabulaire qui est par conséquent le critère prépondérant avec des données similaires excepté pour la mécanique où le pourcentage est nettement plus élevé. La grammaire vient en deuxième position et sa courbe est assez semblable à la précédente avec un pourcentage également plus élevé pour la mécanique. En revanche, les pourcentages sont dans l'ensemble faibles pour l'aspect idiomatique ; l'informatique se différencie légèrement.

\section{Synthèse des commentaires}

Nous avons analysé les résultats chiffrés. Il faut ajouter que trente et une personnes ont noté des remarques et cinq d'entre elles ont joint au questionnaire des documents concernant la rédaction ou l'évaluation. Par conséquent, si l'enquête manquait de finesse, nous comptions sur la question 6 ouverte aux commentaires pour compléter notre étude. Voici une synthèse des points évoqués dans cette rubrique :

Clarté

La clarté est la qualité requise en priorité. Cette remarque revient dix-huit fois. Le message doit être clair, compréhensible après première lecture à vitesse normale, sans ambiguïté et ne nécessitant aucun effort. Par conséquent la langue qui le véhicule et qui contribue à une compréhension aisée doit également avoir cette qualité. Ceci implique que le vocabulaire utilisé est précis et approprié, la phrase bien construite et grammaticalement juste. 4 personnes ont précisé qu'il est impossible de comprendre et 
corriger des phrases présentant des problèmes de structures. On comprend ainsi que les scientifiques anglophones ne tolèrent pas les ambiguïtés et désirent comprendre rapidement car leur temps est précieux.

\section{Tolérance}

Pour huit réviseurs, la rédaction ne doit pas être nécessairement parfaite, un article peut être accepté malgré des erreurs. Une personne note que de nombreux «poor English papers » sont publiés. Une remarque a même été faite à propos du mauvais anglais de certains anglophones. Selon certains réviseurs, il arrive cependant qu'un article soit rejeté à cause de la langue, s'il est réellement incompréhensible ou s'il nécessite trop d'effort. Hormis quelques cas extrêmes, la majorité tolère les erreurs, mais l'un d'entre eux précise que «l'évaluation en réalité dépend essentiellement de la personne et peut varier selon la perception individuelle». Cette remarque est en rapport avec le fait qu'aucune grille d'évaluation de la langue n'est établie et confirme le sentiment que nous avions a priori.

Les critères 6 et 7 n'entrent pas en considération pour 8 autres réviseurs. Il a été spécifié que le contenu scientifique étant suffisamment compliqué, il n'était pas souhaitable de faire intervenir des critères linguistiques complexes. De même un anglais idiomatique n'est pas exigé.

Enfin, huit autres personnes signalent que les articles sont surtout, voire seulement, jugés sur le contenu scientifique.

Donc s'il existe un consensus à propos du premier concept, plus facile à cerner, en revanche, le seuil de tolérance et la notion de «bad » ou " poor English » sont subjectives et recouvrent de multiples interprétations. Sans grille clairement définie, avec des contenus scientifiques extrêmement inégaux, des auteurs ainsi que des environnements dont la notoriété est variable, des domaines plus ou moins porteurs et une compétitivité internationale farouche, dans un tel contexte, le scientifique français aborde le monde de la publication anglophone comme un parcours du combattant. S'il ne maîtrise pas tous les éléments, du moins peut-il éviter la censure pour des raisons linguistiques.

\section{Conditions requises}

Des corrections peuvent être exigées ou conseillées selon les erreurs, des suggestions peuvent être faites afin d'aider les auteurs à améliorer leur anglais, il leur est parfois demandé de faire relire leurs articles par des anglophones. Un mathématicien anglais a cependant précisé qu'il est possible d'exiger des corrections à des auteurs asiatiques ou polonais, mais plus délicat avec les Français de peur de les offenser !

D'autre part, certaines revues imposent un "house style»; dans ce cas les auteurs doivent accepter les contraintes spécifiées. Quelques réviseurs acceptent de corriger euxmêmes des erreurs d'orthographe, de ponctuation, ou rectifient l'ordre des mots dans une phrase lorsque la revue propose ce service (voir question 5). Ils ne corrigent pas les textes trop incorrects en anglais. En revanche, quelques revues précisent dans leur « notes for authors » que les textes en «bad English» ne sont pas tolérés, sans aucune précision complémentaire sur les critères déterminants. Rares sont celles qui acceptent les articles soumis en français hormis les revues canadiennes à cause du bilinguisme de ce pays.

Bilan

Les résultats de cette enquête ont fourni des informations sur deux points essentiels, les critères considérés dans l'évaluation des articles et le processus d'évaluation des articles. 
Il se dégage une tendance générale : les réviseurs exigent clarté et facilité de lecture, ce qui implique une utilisation rigoureuse du vocabulaire avec l'orthographe exacte et une construction grammaticale de la phrase juste. Nous avons relevé cet exemple: «We propose some easy modifications» où "easy» a été remplacé par l'adjectif plus précis « straightforward». L'organisation du discours et l'aspect idiomatique ont une valeur dans l'ensemble toute relative. Ce dernier point est inattendu lorsqu'on sait combien l'anglais de tous les jours est difficile à parfaire précisément à cause du nombre important d'idiotismes.

51 Il est clair que le contenu scientifique prime et que les réviseurs peuvent, grâce à une grille et des consignes précises, évaluer l'intérêt d'un article pour la communauté scientifique. Les critères de qualité de l'anglais ne sont pas spécifiés, mais sont implicitement pris en considération, ce que l'on constate dans les extraits de grilles présentés ci-dessous.
6. COMPREHENSIBILITY:
[] Understood at first reading.
[ ] Several readings required.
[ ] It would take a week to understand this paper.
Presentation:
10. Is the paper arranged in a logical fashion?
11. Is the author's meaning clear?

Sans engager de polémique ni porter de blâme sur ces réviseurs, on peut toutefois s'inquiéter de la part de subjectivité et d'intuition qui intervient alors. Cette enquête prouve implicitement que chacun évalue l'anglais comme il l'entend. Les remarques recueillies à propos de l'absence de points de repère entre l'acceptable et l'inacceptable, de normes, le «flou» qui entoure les appréciations de "poor", «incorrect» ou «

inappropriate », les conditions requises, les « appropriate standards » ou « up to standard » et la conformité au style "maison » confirment notre hypothèse. Dans ce cas, quelles sont les chances d'un scientifique français désireux de publier en pays anglo-saxon, sachant que des articles peuvent être rejetés uniquement pour des raisons linguistiques? Nous n'avons malheureusement aucun moyen de vérifier. Nous osons croire qu'avec un contenu scientifique solide et le respect des contraintes précisées ci-dessus, il réussira à franchir la barrière de la langue et pourra s'imposer comme il le souhaite. Nous conclurons cette enquête avec la réponse de ce mathématicien canadien :

the degree to which a referee considers the linguistic aspects is highly variable and dependent on the referee.

\section{Conclusions et perspectives}

La première enquête menée auprès de scientifiques français devant rédiger des articles en anglais, évoquée dans la première partie, a mis en évidence leurs difficultés majeures : le vocabulaire général et les tournures idiomatiques. À la question sur les difficultés liées au vocabulaire rencontrées lors de la rédaction, 54 sur un total de 61 réponses ont cité comme problème majeur le vocabulaire général. En revanche, leur maîtrise du vocabulaire technique, semble-t-il, leur permet globalement d'utiliser le mot juste. Dans leurs commentaires, ils insistent sur leur manque d'aisance et admettent que leur connaissance de la langue anglaise n'est pas toujours suffisante; ce malaise est alors perçu comme un handicap notoire. Si nous confrontons ces résultats à ceux obtenus auprès des réviseurs anglophones, nous constatons que le vocabulaire à la fois spécialisé 
et général reste l'élément central dans le discours scientifique et nous pensons qu'il devrait faire actuellement l'objet d'une réflexion appropriée de la part des chercheurs français, voire de la mise en place de formations complémentaires spécifiques. D'autre part, les tournures idiomatiques, que $79 \%$ ont considérées comme problème majeur, sujet d'angoisse classique pour des non-anglophones, ne devraient pas a priori discréditer les auteurs puisqu'elles ne paraissent pas indispensables dans ce type de discours.

D'autre part, nombreux sont ceux qui, dans leurs commentaires ou au cours des entretiens, se plaignaient d'avoir été lourdement pénalisés par des anglophones qui ne leur font aucune concession sur la qualité de l'anglais. Les Français ne doivent pas se poser en victimes. À ce propos, il est intéressant de rappeler que des anglophones euxmêmes reçoivent des remarques telles que "Your English must be improved " ou " your paper should be read by English speakers» surtout lorsqu'il s'agit de différences entre l'anglais britannique et l'anglais américain ou que la langue n'est pas suffisamment claire. En effet, les réviseurs américains n'hésitent pas à demander aux auteurs d'américaniser les tournures et l'orthographe britanniques, même lorsque ces derniers sont anglais. On peut aussi être témoin de situations litigieuses auxquelles des anglophones ayant deux adresses ou deux laboratoires de recherche se trouvent confrontés : leur anglais est soit apprécié soit critiqué selon qu'ils sont en Angleterre ou en France.

Il y aurait une certaine contradiction entre la relative tolérance exprimée globalement par les réviseurs et la perception des chercheurs français, à moins que ceux-ci, habitués à l'anglais «de cuisine» des interventions dans le cadre des colloques par exemple, ne fassent preuve de la même négligence pour les publications. Nous avons été amenés, au cours de ces enquêtes, à en lire quelques-unes ; il est certain que les anglophones, voyant leur langue ainsi écorchée, ne peuvent que réagir, sous-entendu qu'ils parviennent à comprendre le message, ce qui n'est pas toujours le cas. Le débat reste ouvert.

Force est de constater que la situation reste complexe. Tant que toutes les revues n'imposeront pas une grille de critères de base d'ordre linguistique, leurs réviseurs auront toute latitude. De même, les auteurs non anglophones, sans directives précises, ne seront pas en mesure de parfaire leur anglais, si, de surcroit, un style "maison » leur impose des normes linguistiques. Cependant, il est peu probable que de telles améliorations soient suffisantes pour offrir de meilleures chances d'accès à la publication en pays anglophone, surtout si les règles du jeu ne sont pas explicitées. Cette enquête n'apportera pas de réponse à ce propos. Un projet d'étude est en cours afin de voir si d'autres facteurs interviennent. De plus, un nouveau courrier a été adressé directement aux revues afin de connaître leur politique en matière de publication et de compléter cette étude.

Nous avons ainsi porté notre attention sur l'un des aspects du monde scientifique, celui de la recherche et de la publication. Cette exploration a été particulièrement intéressante même si les résultats ne sont que partiels. Elle a soulevé le problème de la qualité de la langue utilisée en milieu de non-spécialistes et les questions connexes : définition de la notion de qualité, expression des besoins langagiers, part du subjectif dans l'évaluation, politique globale pour une évaluation homogène et juste, et enfin tolérance à l'égard de productions langagières en langue non maternelle. Certains scientifiques français suggèrent la reconnaissance d'un statut les différenciant des anglophones en leur octroyant par exemple un droit à l'erreur, c'est-à-dire l'acceptation d'un anglais scientifique produit par des non-anglophones. Ce point remettrait notamment en 
question la notion de qualité et de statut de l'anglais comme langue véhiculaire de la communauté scientifique internationale.

Cette étude pourra servir de point de départ à d'autres travaux de recherche: en didactique des langues pour l'enseignement de l'anglais de spécialité et la formation des futurs chercheurs; en travail d'analyse d'erreurs; en analyse stylistique de discours spécialisés grâce à ce "portrait » du langage utilisé dans les publications scientifiques, l'objectif étant une meilleure connaissance et utilisation de l'anglais scientifique.

\section{BIBLIOGRAPHIE}

Crosnier, E. 1990. «L'anglais de spécialité en France : étude des problèmes et besoins des scientifiques et techniciens ». Mémoire de DEA, Université de Toulouse le Mirail.

\section{ANNEXES}

\section{Annexe. Questionnaire}

1. Do you have general guidelines for assessing research papers?

2. If so, are there specific criteria concerning the quality of the English idiom?

3. What are the linguistic criteria taken into account concerning the papers written in English by French scientists?

\begin{tabular}{||l|l|l|l||}
\hline \hline 1. Vocabulary accuracy & ALWAYS & SOMETIMES & NEVER \\
\hline 2. Spelling & & & \\
\hline 3. Grammar accuracy & & & \\
\hline 4. Sentence building & & & \\
\hline 5. Punctuation & & & \\
\hline 6. Sequencing & & & \\
\hline 7. Rhetorical aspect & & & \\
\hline 8. Idlomatic English & & & \\
\hline
\end{tabular}

4. What errors among the above-mentioned points are always unacceptable?

5. Do you have an "Anglicizing" service for correction?

6. Additional personal comments: 


\section{RÉSUMÉS}

Les scientifiques français qui souhaitent publier en pays anglophones sont souvent obligés de revoir la qualité de leur anglais. C'est pourquoi une enquête sous forme de questionnaire a été menée auprès des réviseurs britanniques et américains (referees), qui évaluent les articles scientifiques avant leur publication. L'objectif est d'une part de déterminer les critères linguistiques considérés lors de cette évaluation, d'autre part d'informer sur les processus d'évaluation. Les résultats obtenus peuvent être exploités par les scientifiques afin d'améliorer l'anglais ainsi que par les enseignants pour la formation des futurs chercheurs. Enfin, ils contribuent à une meilleure connaissance de l'anglais scientifique.

French scientists are often required to improve the quality of their English for publishing papers in English-speaking countries. A questionnaire was submitted to British and American referees who review these publications. This survey aims to bring into light the linguistic criteria taken into account and the evaluation process. The results may be used by scientists to improve the English idiom, and for researchers' training. They also contribute to exploring scientific English.

\section{INDEX}

Mots-clés : anglais scientifique, critère d'évaluation, enquête, erreur

Keywords : assessment criteria, error, scientific English, survey

\section{AUTEUR}

\section{ELIZABETH CROSNIER}

INSA, Toulouse. elisabeth.crosnier@wanadoo.fr 